\title{
Satisfacción y experiencia de pacientes en tratamiento con sustitutivos opioides en España. Estudio PREDEPO
}

\section{Patients'satisfaction and experience in treatment with opioid substitution therapy in Spain. The PREDEPO study}

\author{
Francisco Pascual Pastor*, Álvaro Muñoz**, Rodrigo OraA***, Gerardo Flórez****, Pilar \\ Notario*****, Pedro Seijo******, Begoña Gonzalvo*******, Carla Assaf********, Manuel \\ Gómez**, Miguel Ángel Casado**. \\ * Generalitat Valenciana. Unidad de Conductas Adictivas - Departament de Salut d'Alcoi, Alcoi, España. \\ ** Pharmacoeconomics \& Outcomes Research Iberia (PORIB), Madrid, España. \\ *** Red de Salud Mental, IIS Biocruces Bizkaia. CSM Ajuriaguerra Adicciones. RSMB, Bizkaia, España. \\ **** Unidad de Conductas Adictivas de Ourense, Ourense, España. \\ ***** Subdirección General de Adicciones. Centro de Atención a las Adicciones de Latina, Madrid, España. \\ ****** Diputación de Cádiz. Centro de Tratamiento Ambulatorio de Adicciones de Villamartín, Cádiz, España. \\ ******** Red Adicciones, Institut Assistència Sanitària. Departament de Salut Centro de Atención y Seguimiento a las \\ Drogodependencias, Girona, España. \\ ******** Camurus SL, Madrid, España.
}

\section{Resumen}

El objetivo es comparar la satisfacción, experiencia, objetivos y opinión de los pacientes con trastorno por consumo de opioides (TCO) en base a su tratamiento sustitutivo de opioides (TSO) actual (metadona o buprenorfina/naloxona $(\mathrm{B} / \mathrm{N})$ ). El estudio PREDEPO es un estudio observacional, transversal, multicéntrico desarrollado en España que incluyó pacientes adultos, diagnosticados de TCO y en TSO, quienes contestaron una encuesta sobre su tratamiento actual. Se incluyeron 98 pacientes (B/N:50\%, metadona:50\%): edad media de $47 \pm 8$ años y el $80 \%$ varones. A nivel de la satisfacción con su tratamiento, los resultados fueron similares entre grupos. El factor "muy/bastante satisfactorio" que se reportó con mayor frecuencia fue "poder repartir las dosis en varios momentos del día” (44\% B/N vs. $63 \%$ metadona; $\mathrm{p}$ $=, 122)$. Se encontraron diferencias significativas en "tener que recoger la medicación diariamente" donde una menor proporción en el grupo $\mathrm{B} / \mathrm{N}$ contestaron "muy/bastante molesto" versus el grupo metadona ( $19 \%$ vs. $52 \%, p=, 032)$. Los objetivos reportados por la mayoría de los pacientes fueron similares entre grupos ("no sentir más síndrome de abstinencia", "disminuir o dejar definitivamente mi consumo de drogas", "mejorar mi estado de salud" y "dejar de pensar en consumir todos los días") excepto en "no tener más problemas de dinero" (72\% $\mathrm{B} / \mathrm{N}$ vs. 92\% metadona; $\mathrm{p}=, 012)$. Estos resultados evidencian que existen expectativas no cubiertas con los TSO actuales y la necesidad de nuevos tratamientos que disminuyan la carga de la enfermedad, eviten la necesidad de una dosificación diaria y reduzcan el estigma, mejorando así el manejo del paciente, su adherencia y calidad de vida.

Palabras clave: Trastorno por consumo de opioides; satisfacción de pacientes; tratamiento sustitutivo de opioides; metadona, buprenorfina/ naloxona.

\begin{abstract}
The aim of this study was to compare patients' satisfaction, experience, objectives, and opinion based on their current opioid substitution therapy (OST) (buprenorphine/naloxone $(\mathrm{B} / \mathrm{N})$ or methadone). The PREDEPO study is an observational, cross-sectional, multicentric study performed in Spain. Adult patients diagnosed with opioid use disorder (OUD) receiving OST were included. They were asked to fill in a questionnaire regarding their current OST. A total of 98 patients were enrolled (B/N: $50 \%$, methadone: $50 \%)$. Mean age was $47 \pm 8$ years old and $80 \%$ were male. Treatment satisfaction was similar between groups. The most frequently reported factor for being "very/ quite satisfied" was "being able to distribute the dose at different times throughout the day" $(44 \% \mathrm{~B} / \mathrm{N}$ vs. $63 \%$ methadone; $\mathrm{p}=.122)$. A significantly lower proportion of patients in the $\mathrm{B} / \mathrm{N}$ group versus the methadone group reported that having to collect the medication daily was "very/quite annoying" ( $19 \%$ vs. $52 \%, \mathrm{p}=.032)$. Treatment objectives reported by the majority of patients were similar between groups ("not feeling in withdrawal anymore", "reduce/definitely stop drug use", "improve my health", and "stop thinking about using daily") except for "not having money problems anymore" (73\% B/N vs. $92 \%$ methadone; $\mathrm{p}=.012$ ). These results suggest there are several unmet expectations regarding current OST. There is a need for new treatments that reduce the burden of OUD, avoid the need for daily dosing, and are less stigmatizing which in turn could improve patient management, adherence and, quality of life.

Key words: Opioid use disorder; patient satisfaction; opioid substitution therapy; methadone; buprenorphine/naloxone.
\end{abstract}


$\mathrm{E}$ 1 trastorno por consumo de opioides (TCO) es un importante problema de salud crónico y complejo, caracterizado por el uso repetido de opioides y con recaídas frecuentes (American Psychiatric Association, 2013; Dematteis et al., 2017), lo que puede provocar problemas médicos, sociales, y económicos tanto para el individuo como para la sociedad (Canadian Agency for Drugs and Technologies in Health, 2013).

El TCO afecta a millones de personas en todo el mundo (World Health Organization, 2018). En la Unión Europea, en el año 2018, la prevalencia del consumo de opioides de alto riesgo entre adultos se estimó en 1,3 millones de consumidores (Observatorio Europeo de las Drogas y las Toxicomanías, 2020) y se estima que más de 100.000 personas mueren cada año como resultado del uso de opioides (World Health Organization, 2018). En España, la prevalencia del consumo de opioides de alto riesgo entre adultos se estima en torno al $2,2 \%$ por cada 1.000 habitantes (Observatorio Europeo de las Drogas y las Toxicomanías, 2019) y en el año 2017 se contabilizaron más de 1.000 muertes por sobredosis de opioides (Salazar et al., 2020).

El TCO está asociado con una alta tasa de morbimortalidad, siendo las enfermedades infecciosas y los trastornos psiquiátricos las comorbilidades más frecuentes (González-Saiz et al., 2011; Mateu, Astasls y Torrens, 2005; Roncero et al., 2016). Muchos consumidores de opioides a largo plazo en Europa practican el policonsumo, por lo que el TCO también se asocia a problemas relacionados con el consumo de drogas como la sobredosis, delincuencia, desempleo, la exclusión y desventajas sociales, entre otros (Observatorio Europeo de las Drogas y las Toxicomanías, 2020).

Superar la adicción y reinsertarse en la sociedad suele requerir tratamiento a largo plazo (Dematteis et al., 2017; Observatorio Europeo de las Drogas y las Toxicomanías, 2020; Observatorio Vasco de Drogodependencias, 2004; Roncero et al., 2017). Aunque hay tratamientos para el TCO basados en la abstinencia, se recomienda la farmacoterapia con tratamientos sustitutivos de opioides (TSO) y apoyo psicosocial (Dematteis et al., 2017), siendo la metadona y la combinación de buprenorfina y naloxona (antagonista) (B/N) sublingual los TSO más utilizados en España (Roncero et al., 2015). Ambos tratamientos reducen el deseo de consumo de opioides, permitiendo un mejor control de las comorbilidades psiquiátricas y orgánicas. Además, se asocian con una reducción en la tasa de enfermedades infecciosas y hospitalizaciones, aumentando la supervivencia en general (Koehl, Zimmerman y Bridgeman, 2019; Sordo et al., 2017; Volkow, Frieden, Hyde y Cha, 2014).

Sin embargo, la falta de adherencia y el abandono del tratamiento son habituales (Calvo et al., 2018; Strang et al., 2017), existiendo un porcentaje elevado de pacientes que reinician el tratamiento (Observatorio Europeo de las Drogas y las Toxicomanías, 2019). Tanto la metadona como la $\mathrm{B} / \mathrm{N}$ tienen una posología diaria y requieren visitas frecuentes por parte de los pacientes a las unidades de conductas adictivas o servicios de farmacia para la recogida de la medicación. Para muchos pacientes es un proceso estigmatizante, que limita su libertad y calidad de vida, dificultando la actividad laboral, las vacaciones, así como otras actividades cotidianas. Es por esto por lo que cada vez más pacientes con TSO reclaman mayor tiempo libre para poder dedicarlo a normalizar su vida (Harris y McElrath, 2012; Treloar y Valentine, 2013). Es importante tener en cuenta tanto las condiciones de los pacientes, como su satisfacción y/o experiencias a la hora de instaurar un tratamiento, con el objetivo de adecuar la mejor estrategia farmacológica. En este sentido, cada vez cobran mayor importancia la evaluación de las medidas de experiencia percibidas por los pacientes (Patient Reported Experiences Measures, PREM). Los PREM, evalúan la percepción, satisfacción y/o experiencia de los pacientes sobre la atención, los tratamientos y el soporte recibido. Siendo, por tanto, un indicador de calidad de la asistencia recibida y un elemento imprescindible para la medicina y la investigación centrada en los pacientes (Alonso-Caballero y Ferrer-Forés, 2017).

La satisfacción con el tratamiento y con la atención médica recibida es el grado en que la experiencia de un paciente es comparable a sus expectativas (Iftikhar et al., 2011). Conocer el grado de satisfacción de los pacientes es un indicador, cada vez más empleado, para lograr el éxito terapéutico y mejorar la calidad de vida de los pacientes (Florek, Wang y Armstrong, 2018). Por ello, el objetivo de este estudio fue comparar la satisfacción, experiencia, objetivos y opinión de los pacientes con TCO en base a su TSO actual (metadona o B/N)

\section{Método}

\section{Diseño del estudio}

El estudio PREDEPO es un estudio observacional, transversal y multicéntrico que se llevó a cabo en 6 centros de atención a adicciones, de 6 Comunidades Autónomas de España, pertenecientes al Sistema Nacional de Salud. Este estudio fue aprobado por el Comité de Ética de la Investigación con medicamentos de Euskadi (CEIm-E) (País Vasco, España) y se realizó de acuerdo con los principios de la Declaración de Helsinki relativa a la investigación médica en seres humanos.

\section{Población de estudio}

Se reclutaron pacientes adultos diagnosticados con TCO mediante el Manual Diagnóstico y Estadístico de los trastornos mentales (DSM-5) (American Psychiatric Association, 2013), en tratamiento con TSO (con prescripción médica) para TCO y que proporcionaron su consentimiento infor- 
mado por escrito. No se incluyeron pacientes con incapacidad para leer y/o comprender las preguntas del diario del paciente o el consentimiento informado, así como aquellos pacientes con disfunción psíquica evidente que pudiese conllevar una falta de disposición o incapacidad del paciente para cumplir con los procedimientos del estudio.

\section{Recogida de datos}

Para evitar el sesgo en la recogida de información, el reclutamiento de los pacientes se realizó según acudieron a consulta de forma consecutiva hasta completar cada grupo de tratamiento. La recogida de datos se realizó entre septiembre y octubre de 2020. Los investigadores de cada centro cumplimentaron un cuaderno de recogida de datos (CRD) de forma retrospectiva con datos sociodemográficos y clínicos obtenidos a partir de las historias clínicas y registros informáticos del centro. Por otro lado, los pacientes cumplimentaron de forma transversal un diario del paciente donde se incluyeron preguntas para conocer su opinión y experiencia acerca de su TSO.

\section{Diseño del CRD y del diario del paciente}

Un Comité Científico formado por el presidente de la Sociedad Científica Española de Estudios sobre el Alcohol, el Alcoholismo y las otras Toxicomanías (SOCIDROGALCOHOL), un experto en psicología de la salud, gestión y evaluación y un paciente con TCO colaboraron con dos miembros expertos en Investigación de Resultados en Salud para el diseño del CRD y del diario del paciente. Además, el paciente con TCO participó en la adaptación del lenguaje de las preguntas del diario del paciente para facilitar su comprensión para los participantes.

El diario del paciente estaba compuesto por preguntas de opciones fijas y varias escalas de Likert. Para conocer los objetivos de los pacientes se empleó una Escala Likert gradual con 4 opciones de respuesta, agrupándose los resultados según el grado de conformidad ["para nada" y "no del todo" vs. "sí, bastante" y "sí, absolutamente"]. Para conocer la satisfacción de los pacientes con posibles situaciones asociadas a su TSO en el momento del estudio, se empleó una escala Likert gradual con 5 opciones de respuesta, agrupándose los resultados según el grado de satisfacción ["muy molesto" y "bastante molesto" vs. "ni molesto ni satisfactorio" vs. "bastante satisfactorio" y "muy satisfactorio"]. Para conocer el grado de satisfacción de los pacientes por la atención de los profesionales recibida, así como con el TSO en el momento del estudio y la valoración de su eficacia se emplearon escalas Likert continuas del 1-10, siendo 1 el valor menos positivo y 10 el más positivo.

\section{Objetivos del estudio}

El objetivo principal de este estudio fue comparar la satisfacción y experiencia de los pacientes con TCO en base a su TSO actual (metadona o B/N). Los objetivos secun- darios fueron determinar, en función del grupo de tratamiento, los objetivos y opiniones de los pacientes, así como sus características sociodemográficas y clínicas.

\section{Tamaño muestral}

Los resultados del presente artículo han sido obtenidos de una extensión al análisis principal del estudio PREDEPO por lo que se ha obtenido de dicho estudio toda la metodología, incluyendo diseño, variables incluidas y el tamaño de muestra. Para dicho análisis principal, se calculó el tamaño muestral necesario para estimar, con una precisión del $10 \%$ y una confianza del $95 \%$, el porcentaje de pacientes que estarían dispuestos a cambiar de tratamiento. Esta estimación está basada en el estudio de Bendimerad, Kosim y Trojak (2019) en el que el 53,2\% de los pacientes en tratamiento para el TCO estuvieron dispuestos a cambiar o modificar su tratamiento. Según estos datos se precisarían 96 pacientes, a los que se le añadirían un $10 \%$ adicional por posibles pérdidas en el estudio, llegando hasta un total de 106 pacientes.

\section{Análisis estadístico}

Para el estudio descriptivo se calcularon frecuencias absolutas y relativas en variables cualitativas, así como media y desviación estándar en variables cuantitativas y el intervalo de confianza al 95\% ( $\mathrm{IC}=95 \%)$ para el resultado del objetivo principal. Para el análisis inferencial que comparaba las características de los pacientes según hubieran recibido metadona o B/N se emplearon las pruebas de ji cuadrado de Pearson y la Prueba exacta de Fisher en caso de incumplirse algún requisito de la anterior en variables cualitativas y la prueba $\mathrm{T}$ de Student en variables cuantitativas, asumiendo un supuesto de normalidad al pasar de 30 casos en cada grupo. El umbral de error tipo I empleado para aceptar o rechazar las hipótesis nulas fue del 5\%. Los cálculos se realizaron con el software R 4.0.4.

\section{Resultados}

\section{Población de estudio}

Noventa y nueve pacientes fueron reclutados entre los 6 centros participantes, de los que se analizaron 98 pacientes (50\% en tratamiento con metadona y el $50 \%$ restante con $\mathrm{B} / \mathrm{N}$ ). Un paciente en tratamiento con naltrexona fue excluido del análisis por incumplimiento de los criterios de selección del estudio.

\section{Características sociodemográficas y clínicas}

En la tabla 1 y 2 se muestran, respectivamente, las características sociodemográficas y clínicas de los pacientes incluidos en el análisis del estudio. La edad media de los pacientes fue $46,9 \pm 8,4$ años y el 79,6\% de los participantes fueron hombres. Se observó un número significativamente menor de infecciones por VHC en los pacientes con B/N, 
Tabla 1. Características sociodemográficas.

\begin{tabular}{|c|c|c|c|c|}
\hline Variable & $\begin{array}{l}\text { TOTAL } \\
(n=98)\end{array}$ & $\begin{array}{c}\text { Metadona } \\
(n=49)\end{array}$ & $\begin{array}{c}B / N \\
(n=49)\end{array}$ & $p$-valo \\
\hline \multicolumn{5}{|l|}{ Edad, años } \\
\hline Media (DE) & $46,9(8,4)$ & $46,7(7,7)$ & $47,0(9,2)$ & ,873 \\
\hline \multicolumn{5}{|l|}{ Sexo, n (\%) } \\
\hline Masculino & $78(79,6)$ & $40(81,6)$ & $38(77,6)$ & \multirow{2}{*}{, 616} \\
\hline Femenino & $20(21,4)$ & $9(18,4)$ & $11(22,4)$ & \\
\hline \multicolumn{5}{|l|}{ Estado civil, n (\%) } \\
\hline Soltero/a & $49(50,0)$ & $26(53,1)$ & $23(46,9)$ & \multirow{3}{*}{, 576} \\
\hline Casado/a o en pareja & $31(31,6)$ & $16(32,6)$ & $15(30,6)$ & \\
\hline Separado/a o divorciado/a & $18(18,4)$ & $7(14,3)$ & $11(22,5)$ & \\
\hline \multicolumn{5}{|l|}{ Situación familiar estable, n (\%) } \\
\hline Tiene hijos & $37(37,8)$ & $13(26,5)$ & $24(49,0)$ & ,022 \\
\hline Situación estable en la vivienda & $70(71,4)$ & $36(73,5)$ & $34(69,4)$ & ,655 \\
\hline \multicolumn{5}{|l|}{ Convivencia, n (\%) } \\
\hline Familia de origen & $36(36,7)$ & $18(36,7)$ & $18(36,7)$ & \multirow{5}{*}{,898 } \\
\hline Pareja & $29(29,6)$ & $16(32,7)$ & $13(26,5)$ & \\
\hline Solo & $27(27,6)$ & $13(26,6)$ & $14(28,7)$ & \\
\hline Piso compartido & $2(2,0)$ & $1(2,0)$ & $1(2,0)$ & \\
\hline Otra & $4(4,1)$ & $1(2,0)$ & $3(6,1)$ & \\
\hline \multicolumn{5}{|l|}{ Nivel de estudios, $n$ (\%) } \\
\hline Estudios primarios & $54(55,1)$ & $31(63,3)$ & $23(46,9)$ & \multirow{4}{*}{,333 } \\
\hline Estudios secundarios & $34(34,7)$ & $13(26,5)$ & $21(42,9)$ & \\
\hline Sin estudios & $7(7,1)$ & $4(8,2)$ & $3(6,1)$ & \\
\hline Estudios Universitarios & $3(3,1)$ & $1(2,0)$ & $2(4,1)$ & \\
\hline \multicolumn{5}{|l|}{ Situación laboral, n (\%) } \\
\hline Con prestación social & $48(49,0)$ & $24(49,0)$ & $24(49,0)$ & \multirow{5}{*}{,666 } \\
\hline Parado & $22(22,4)$ & $13(26,5)$ & $9(18,4)$ & \\
\hline Activo & $21(21,4)$ & $9(18,4)$ & $12(24,5)$ & \\
\hline Trabajo no remunerado & $3(3,1)$ & $2(4,1)$ & $1(2,0)$ & \\
\hline Otra & $4(4,1)$ & $1(2,0)$ & $3(6,1)$ & \\
\hline
\end{tabular}

Nota. B/N: buprenorfina/naloxona; DE: desviación estándar.

en comparación con los pacientes con metadona: $(2,04 \%$ con $\mathrm{B} / \mathrm{N}$ vs. $22,45 \%$ con metadona; $\mathrm{p}=, 002)$. Además, se observó un porcentaje significativamente mayor de pacientes con hijos en el grupo con $\mathrm{B} / \mathrm{N}$, en comparación con el grupo con metadona $(50,0 \%$ con $\mathrm{B} / \mathrm{N}$ vs. $26,5 \%$ con metadona; $\mathrm{p}=$,022). También se observaron diferencias estadísticamente significativas relacionadas con los años en tratamiento para el TCO $(10,4 \pm 7,6$ años con B/N vs. 16,7 $\pm 9,3$ años con metadona; $\mathrm{p}<, 001)$ y en el inicio del TSO en el momento del estudio $(4,6 \pm 4,2$ años con $\mathrm{B} / \mathrm{N}$ vs. 9,7 $\pm 8,6$ años con metadona; $\mathrm{p}<, 001$ ), así como en la frecuencia en la recogida de la medicación $(\mathrm{p}<, 001)$, donde una mayor proporción de pacientes con metadona recogían la medicación con una frecuencia menor a un mes $(89,8 \%)$ en comparación con los pacientes con B/N (23,40\%), donde la mayoría de los pacientes $(42,6 \%)$ recogía la mediación mensualmente (Figura 1).

\section{Satisfacción de los pacientes con su TSO}

En la figura 2 se muestran los resultados de la satisfacción de los pacientes con posibles situaciones asociadas a su TSO. El factor que se reportó con mayor frecuencia en ambos grupos de tratamiento como "muy o bastante satisfactorio" fue el de "poder repartir las dosis en varios momentos del día” $(44,4 \%, 12 / 27$ con B/N vs. 63,0\%, 17/27 con metadona; $\mathrm{p}=, 122)$. Los factores que los pacientes reportaron de forma más frecuente como "muy molesto" o "bastante molesto" en el grupo de B/N fue "ser capaz de vez en cuando de no tomar la medicación para poder usar sustancias ilícitas en otros lugares", "poder vender el tratamiento" y "sentirse avergonzado/estigmatizado al tener supervisada diariamente la administración de mi tratamiento". Por otro lado, el factor que los pacientes reportaron de forma más frecuente como "muy molesto" o "bastante molesto" en el grupo de metadona fue "tener que recoger la medicación diariamente", seguido de "tener que recoger con frecuencia (diariamente, semanalmente) su tratamiento" y de "sentirse avergonzado/estigmatizado al tener supervisada diariamente la administración de mi tratamiento". La única variable donde se encontraron diferencias significativas entre grupos fue en la de "tener que recoger la medicación diariamente" donde se observó una menor proporción de pacientes en el grupo de $\mathrm{B} / \mathrm{N}$ que contestaron "muy molesto" o "bastante molesto" versus el 
Francisco Pascual Pastor, Álvaro Muñoz, Rodrigo Oraa, Gerardo Flórez, Pilar Notario, Pedro Seijo, Begoña Gonzalvo, Carla Assaf, Manuel Gómez, Miguel Ángel Casado

Tabla 2. Características clínicas.

\begin{tabular}{|c|c|c|c|c|}
\hline Variable & $\begin{array}{l}\text { TOTAL } \\
(\mathrm{n}=98)\end{array}$ & $\begin{array}{c}\text { Metadona } \\
(n=49)\end{array}$ & $\begin{array}{c}B / N \\
(n=49)\end{array}$ & $p$-valor \\
\hline \multicolumn{5}{|l|}{ Inicio de consumo de opioides, años } \\
\hline Media (DE) & $21,4(11,5)$ & $23,1(11,2)$ & $19,6(11,6)$ & \multirow{2}{*}{,148 } \\
\hline Valores perdidos & 7 & 4 & 3 & \\
\hline \multicolumn{5}{|l|}{ Inicio del primer TSO, años } \\
\hline Media (DE) & $13,5(9,0)$ & $16,7(9,3)$ & $10,4(7,6)$ & $<, 001$ \\
\hline \multicolumn{5}{|l|}{ Inicio del TSO actual, años } \\
\hline Media (DE) & $7,3(7,2)$ & $9,7(8,6)$ & $4,6(4,2)$ & $<, 001$ \\
\hline \multicolumn{5}{|l|}{ № de tratamientos previos, $\mathrm{n}(\%)$} \\
\hline 1 o más & $56(57,1)$ & $25(51,0)$ & $31(63,3)$ & \multirow{2}{*}{,640 } \\
\hline Ninguno & $42(42,9)$ & $24(49,0)$ & $18(36,7)$ & \\
\hline \multicolumn{5}{|l|}{ Fase actual del TSO, $n$ (\%) } \\
\hline Mantenimiento & $87(88,8)$ & $45(91,8)$ & $42(85,7)$ & \multirow{2}{*}{,337 } \\
\hline Reducción & $11(11,2)$ & $4(8,2)$ & $7(14,3)$ & \\
\hline \multicolumn{5}{|c|}{ Cambios de posología en el último año, $n$ (\%) } \\
\hline No & $70(71,4)$ & $32(65,3)$ & $38(77,6)$ & \multirow{2}{*}{180} \\
\hline Sí & $28(28,6)$ & $17(34,7)$ & $11(22,4)$ & \\
\hline \multicolumn{5}{|l|}{ Adicción a otras sustancias, $n$ (\%) } \\
\hline Sin adicción & $11(11,2)$ & $3(6,1)$ & $8(16,3)$ & \multirow{2}{*}{,110 } \\
\hline Adicción a 1 o más sustancias & $87(88,8)$ & $46(93,9)$ & $41(83,7)$ & \\
\hline \multicolumn{5}{|l|}{ Sustancias, $\mathrm{n}(\%)^{\#}$} \\
\hline Tabaco & $72(73,5)$ & $39(79,6)$ & $33(67,4)$ & ,170 \\
\hline Cocaína & $43(43,9)$ & $26(53,1)$ & $17(34,7)$ & 067 \\
\hline Cannabis & $21(21,4)$ & $13(26,5)$ & $8(16,3)$ &, 218 \\
\hline Ansiolíticos & $15(15,3)$ & $9(18,4)$ & $6(12,2)$ &, 400 \\
\hline Alcohol & $14(14,3)$ & $6(12,2)$ & $8(16,3)$ &, 564 \\
\hline Estimulantes & $6(6,1)$ & $3(6,1)$ & $3(6,1)$ & 1,000 \\
\hline \multicolumn{5}{|l|}{ Patología orgánica, n (\%) ${ }^{*}$} \\
\hline $\mathrm{VIH}$ & $19(19,3)$ & $9(18,4)$ & $10(20,4)$ & ,798 \\
\hline VHC & $12(12,2)$ & $11(22,5)$ & $1(2,0)$ & ,002 \\
\hline Trastorno pulmonar & $9(9,2)$ & $5(10,2)$ & $4(8,2)$ & 1,000 \\
\hline VHB & $6(6,1)$ & $5(10,2)$ & $1(2,0)$ & ,111 \\
\hline Enfermedad cardiaca & $1(1,0)$ & $0(0,0)$ & $1(2,0)$ & 1,000 \\
\hline Otra & $10(10,2)$ & $4(8,2)$ & $6(12,2)$ & ,487 \\
\hline \multicolumn{5}{|c|}{ Comorbilidades psiquiátricas, $\mathrm{n}(\%)^{\#}$} \\
\hline Trastorno de personalidad & $26(26,5)$ & $13(26,5)$ & $13(26,5)$ & 1,000 \\
\hline Depresión mayor & $18(18,4)$ & $6(12,2)$ & $12(24,5)$ & ,118 \\
\hline Psicosis / esquizofrenia & $14(14,3)$ & $5(10,2)$ & $9(18,4)$ &, 248 \\
\hline TDAH & $5(5,1)$ & $4(8,2)$ & $1(2,0)$ & ,362 \\
\hline Otra & $7(7,1)$ & $4(8,2)$ & $3(6,1)$ & 1,000 \\
\hline \multicolumn{5}{|l|}{ Otros tratamientos, $\mathrm{n}(\%)$} \\
\hline Sin tratamiento & $26(26,5)$ & $12(24,5)$ & $14(28,6)$ & \multirow{2}{*}{,647 } \\
\hline Con 1 o más tratamientos & $72(73,5)$ & $37(75,5)$ & $35(71,4)$ & \\
\hline \multicolumn{5}{|l|}{ Tratamientos, $\mathbf{n}(\%)^{\#}$} \\
\hline Ansiolíticos/hipnóticos & $57(58,6)$ & $31(63,3)$ & $26(53,1)$ & ,306 \\
\hline Antidepresivos & $32(32,7)$ & $15(30,6)$ & $17(34,7)$ & 667 \\
\hline Antipsicóticos & $25(25,5)$ & $12(24,5)$ & $13(26,5)$ &, 817 \\
\hline Otros & $10(10,2)$ & $4(8,2)$ & $6(12,2)$ & ,487 \\
\hline
\end{tabular}

Nota. "Los pacientes podían presentar más de una opción.

B/N: buprenorfina/naloxona; DE: desviación estándar; TDAH: trastorno por déficit de atención e hiperactividad; TSO, tratamiento sustitutivo de opioides; VHB: virus de la hepatitis B; VHC: virus de la hepatitis C; VIH: virus de la inmunodeficiencia humana.

grupo de metadona $(19,0 \%, 4 / 21$ vs. $52,2 \%, 12 / 23 \mathrm{p}=$ ,032, respectivamente).

En ambos grupos de tratamiento, los pacientes indicaron una puntuación mayor de 8/10 al preguntarles por la satisfacción con su TSO en el momento del estudio $(8,9 / 10$ con $\mathrm{B} / \mathrm{N}$ vs. $8,4 / 10$ con metadona; $\mathrm{p}=, 107)$. Los pacientes con $\mathrm{B} / \mathrm{N}$ evaluaron de forma más positiva que los pacientes con metadona tanto la atención recibida por 

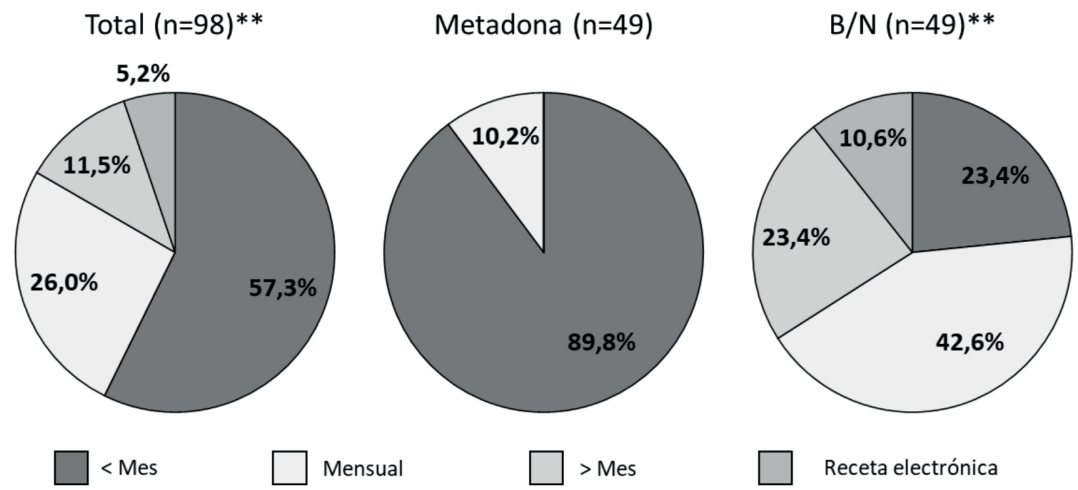

Nota. ${ }^{*} 2$ valores perdidos. $\mathrm{B} / \mathrm{N}$ : buprenorfina/naloxona. Comparación entre grupos de tratamiento: $p$-valor $<$, oo1. Comparación entre grupos y frecuencia mensual y <de un mes: $p$-valor $<0,001$.

Figura 1. Frecuencia de recogida de la medicación.

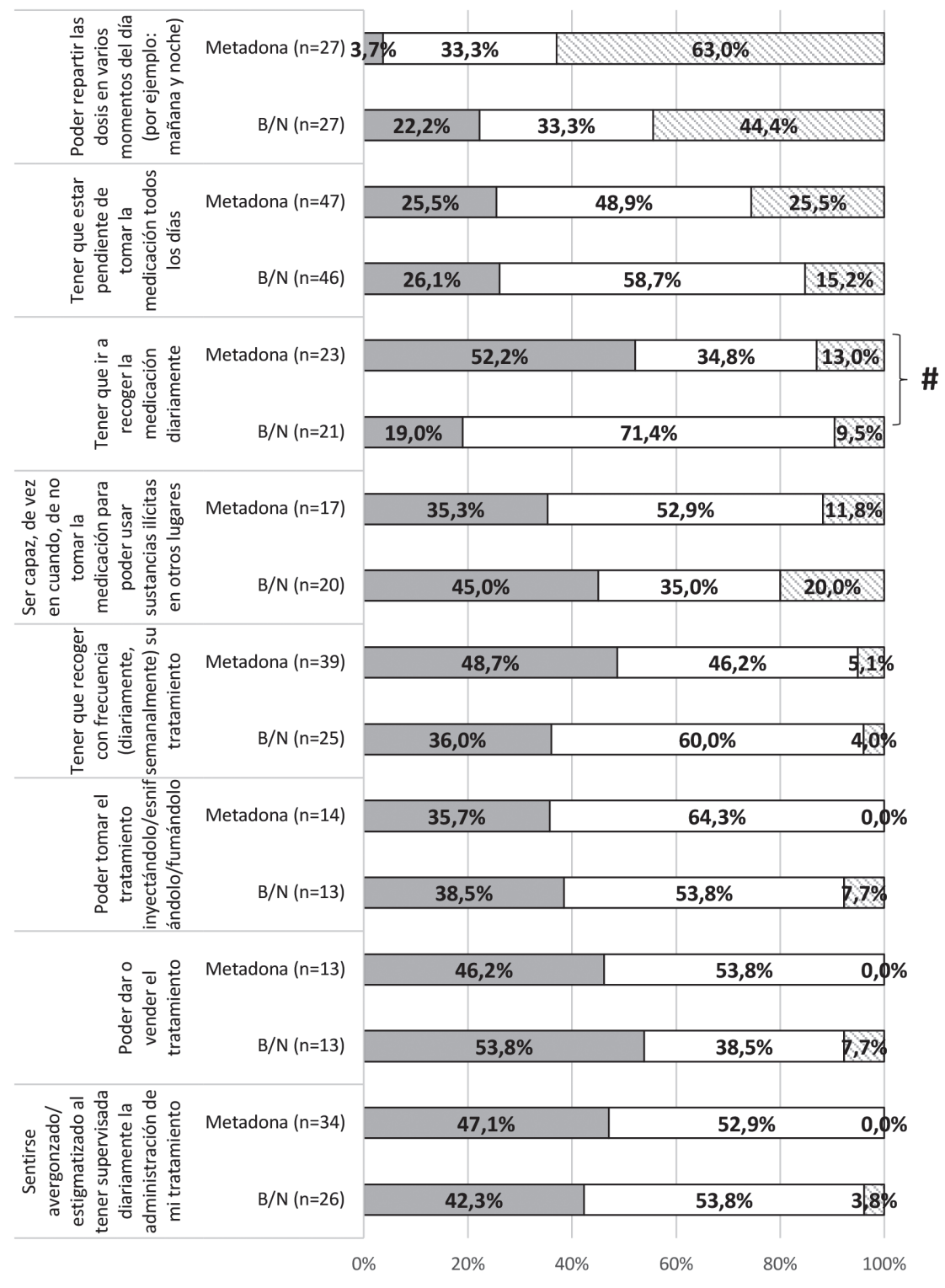

$\square$ Muy o bastante molesto $\square$ Ni molesto ni satisfactorio $\square$ Muy o bastante satisfactorio

Nota. ${ }^{\#} \mathrm{p}=, 032 . \mathrm{B} / \mathrm{N}$ : buprenorfina/naloxona; TSO, tratamiento sustitutivo de opioides.

Figura 2. Satisfacción de los pacientes con su TSO. 
los profesionales sanitarios $(9,2 / 10$ con $\mathrm{B} / \mathrm{N}$ vs. $8,7 / 10$ con metadona; $\mathrm{p}=, 048)$, como la valoración de la eficacia del TSO en el momento del estudio $(9,2 / 10$ con $\mathrm{B} / \mathrm{N}$ vs. $8,5 / 10$ con metadona; $\mathrm{p}=, 014$ ) (Tabla 3 ).

\section{Experiencia de los pacientes con su TSO}

No se encontraron diferencias estadísticamente significativas entre grupos de tratamiento al analizar las experiencias de los pacientes con su tratamiento, aunque casi el doble de pacientes con metadona indicó encontrarse en circunstancias/condiciones que dificultaron la toma de su tratamiento ( $14,9 \%$ con B/N vs. $28,6 \%$ con metadona). La mayoría de los pacientes en ambos grupos de tratamiento indicaron no olvidarse de tomar la medicación o hacerlo rara vez $(77,1 \%$ con $\mathrm{B} / \mathrm{N}$ vs. $75,5 \%$ con metadona), y tomar siempre la medicación prescrita $(79,6 \%$ con $\mathrm{B} / \mathrm{N}$ vs. $89,8 \%$ con metadona) así como también la dosis prescrita. Sin embargo, un $22,5 \%$ y un $10,2 \%$ de pacientes con B/N y metadona, respectivamente, indicaron que guardan la do- sis que no toman a diario para poder disponer de reservas para su consumo personal (Tabla 3 ).

\section{Objetivos de los pacientes}

El 80,6\% del total de pacientes indicó que desearía dejar todo tipo de consumo de opioides, incluido el TSO $(73,5 \%$ con $\mathrm{B} / \mathrm{N}$ vs. $87,8 \%$ con metadona; $\mathrm{p}=, 109)$ (datos no mostrados). No se observaron diferencias significativas entre grupos al analizar los objetivos de los pacientes con respecto a su TSO, excepto en "no tener más problemas de dinero" $(72,3 \%$ con $\mathrm{B} / \mathrm{N}$ vs. 91,8 con metadona; $\mathrm{p}=$ ,012). Los objetivos reportados por la mayoría ( 90\%) de los pacientes fueron similares entre grupos: "no sentir más síndrome de abstinencia" (97,9\% con $\mathrm{B} / \mathrm{N}$ vs. 93,9 con metadona), "disminuir o dejar definitivamente mi consumo de drogas" (91,7\% con B/N vs. 93,9 con metadona), "mejorar mi estado de salud" $(89,6 \%$ con $\mathrm{B} / \mathrm{N}$ vs. $91,8 \%$ con metadona) y "dejar de pensar en consumir todos los días" (89,6\% con B/N vs. 91,8\% con metadona) (Figura 3).

Tabla 3. Experiencia y satisfacción de los pacientes con su TSO.

\begin{tabular}{|c|c|c|c|c|}
\hline Variable & $\begin{array}{l}\text { TOTAL } \\
(n=98)\end{array}$ & $\begin{array}{c}\text { Metadona } \\
(\mathrm{n}=49)\end{array}$ & $\begin{array}{c}B / N \\
(n=49)\end{array}$ & p-valor \\
\hline \multicolumn{5}{|l|}{ ¿Olvida tomar su medicación?, n (\%) } \\
\hline No, nunca o raras veces & $74(76,3)$ & $37(75,5)$ & $37(77,1)$ & \multirow{2}{*}{,855 } \\
\hline Si, a veces o a menudo & $23(23,7)$ & $12(24,5)$ & $11(22,9)$ & \\
\hline Valor perdido & 1 & 0 & 1 & \\
\hline \multicolumn{5}{|c|}{ ¿Se ha encontrado en circunstancias/condiciones donde le ha sido difícil o poco práctico tomar su tratamiento?, n (\%) } \\
\hline No, nunca o raras veces & $75(78,1)$ & $35(71,4)$ & $40(85,1)$ & \multirow{2}{*}{, 105} \\
\hline $\mathrm{Si}$, a veces o a menudo & $21(21,9)$ & $14(28,6)$ & $7(14,9)$ & \\
\hline Valor perdido & 2 & 0 & 2 & \\
\hline \multicolumn{5}{|l|}{ ¿Toma la medicación prescrita?, n (\%) } \\
\hline No, nunca & $1(1,0)$ & $1(2,0)$ & $0(0,00)$ & \multirow{4}{*}{, 273} \\
\hline Sí, a veces & $3(3,1)$ & $1(2,0)$ & $2(4,1)$ & \\
\hline Sí, a menudo & $11(11,2)$ & $3(6,1)$ & $8(16,3)$ & \\
\hline Sí, siempre & $83(84,7)$ & $44(89,8)$ & $39(79,6)$ & \\
\hline \multicolumn{5}{|l|}{ ¿Qué hace con la medicación que no toma a diario?, n (\%) } \\
\hline Me la guardo porque prefiero tener reservas para mi consumo personal & $16(16,3)$ & $5(10,2)$ & $11(22,4)$ & \multirow{5}{*}{, 210} \\
\hline La vendo de vez en cuando o regularmente & $1(1,0)$ & $1(2,0)$ & $0(0,0)$ & \\
\hline Prefiero guardar algunas de reserva para poder ayudar a alguien & $3(3,1)$ & $1(2,0)$ & $2(4,1)$ & \\
\hline No aplica porque tomo siempre la dosis de medicación que me indican & $76(77,6)$ & $40(81,6)$ & $36(73,5)$ & \\
\hline Otra & $2(2,0)$ & $2(4,1)$ & $0(0,0)$ & \\
\hline \multicolumn{5}{|l|}{ ¿Toma la medicación en una vez o la reparte?, n (\%) } \\
\hline Siempre reparto la medicación a lo largo del día & $20(20,4)$ & $13(26,5)$ & $7(14,3)$ & \multirow{5}{*}{, 579} \\
\hline A menudo reparto la medicación a lo largo del día & $5(5,1)$ & $2(4,1)$ & $3(6,1)$ & \\
\hline A veces reparto la medicación a lo largo del día & $3(3,1)$ & $2(4,1)$ & $1(2,0)$ & \\
\hline Raras veces reparto la medicación a lo largo del día & $4(4,1)$ & $2(4,1)$ & $2(4,1)$ & \\
\hline Siempre tomo la medicación en una sola vez & $66(67,3)$ & $30(61,2)$ & $36(73,5)$ & \\
\hline \multicolumn{5}{|l|}{ Satisfacción con la atención de los profesionales* } \\
\hline Media (DE) & $8,9(1,3)$ & $8,7(1,5)$ & $9,2(1,0)$ & ,048 \\
\hline \multicolumn{5}{|l|}{ Satisfacción con el TSO actual* } \\
\hline Media (DE) & $8,6(1,4)$ & $8,4(1,5)$ & $8,9(1,3)$ &, 107 \\
\hline \multicolumn{5}{|l|}{ Valoración de la eficacia del TSO actual* } \\
\hline Media (DE) & $8,9(1,5)$ & $8,5(1,7)$ & $9,2(1,1)$ &, 014 \\
\hline
\end{tabular}

Nota. ${ }^{*}$ Escala Likert de valoración del 1 al 10 , siendo $1=$ más negativo y 10 = más positivo.

$\mathrm{B} / \mathrm{N}$ : buprenorfina/naloxona; DE: desviación estándar; TSO, tratamiento sustitutivo de opioides. 


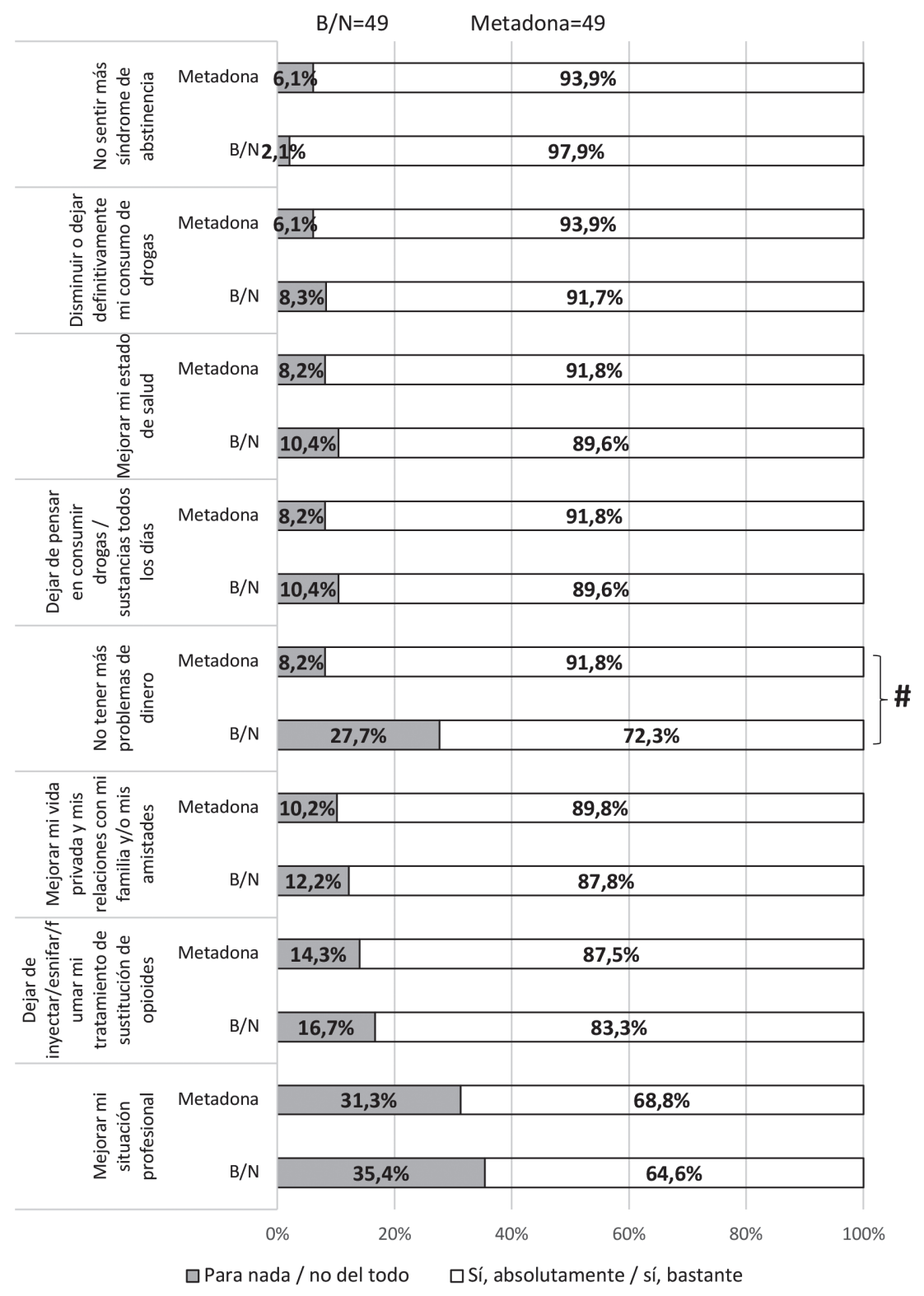

Nota. ${ }^{\#} \mathrm{p}=, 012 ; \mathrm{B} / \mathrm{N}$ : buprenorfina/naloxona; TSO, tratamiento sustitutivo de opioides.

Figura 3. Objetivos de los pacientes con su TSO.

\section{Opinión de los pacientes sobre su TSO}

Las opiniones de los pacientes sobre su sobre su tratamiento se muestran en la figura 4. En ambos grupos de tratamiento la mayor parte de los pacientes indicó que con su tratamiento no se produjo un aumento en el consumo de alcohol $(93,9 \%$ con $\mathrm{B} / \mathrm{N}$ vs. $100 \%$ con metadona; $\mathrm{p}=$ ,106), ni de sustancias ilegales $(95,6 \%$ con $\mathrm{B} / \mathrm{N}$ vs. $91,8 \%$ con metadona; $\mathrm{p}=, 679)$, ni de otros fármacos $(91,1 \%$ con $\mathrm{B} / \mathrm{N}$ vs. $83,7 \%$ con metadona; $\mathrm{p}=, 280)$ y que no tienen ganas de seguir consumiendo $(93,2 \%$ con $\mathrm{B} / \mathrm{N}$ vs. $87,7 \%$ con metadona; $\mathrm{p}=, 492)$. Sólo se observaron diferencias significativas entre grupos de tratamiento al preguntar a los pacientes si consideraban su dosis inferior a sus necesidades. Aproximadamente, 3 veces más de pacientes con metadona consideraron que su dosis era inferior a sus necesidades, en comparación con los pacientes con $\mathrm{B} / \mathrm{N}$ $(13,3 \%$ con $\mathrm{B} / \mathrm{N}$ vs. $34,7 \%$ con metadona; $\mathrm{p}=, 016)$.

\section{Discusión}

Este estudio reporta resultados interesantes en relación con la satisfacción con el tratamiento reportada por los pacientes. Aspectos como la frecuencia diaria de la recogida de la medicación, el estigma, la posibilidad de consumir opioides ilegales durante el tratamiento y el poder vender la medicación fueron los motivos de insatisfacción con el tratamiento actual reportados con mayor frecuencia por parte de ambos grupos de pacientes (metadona y B/N). Por otro lado, 


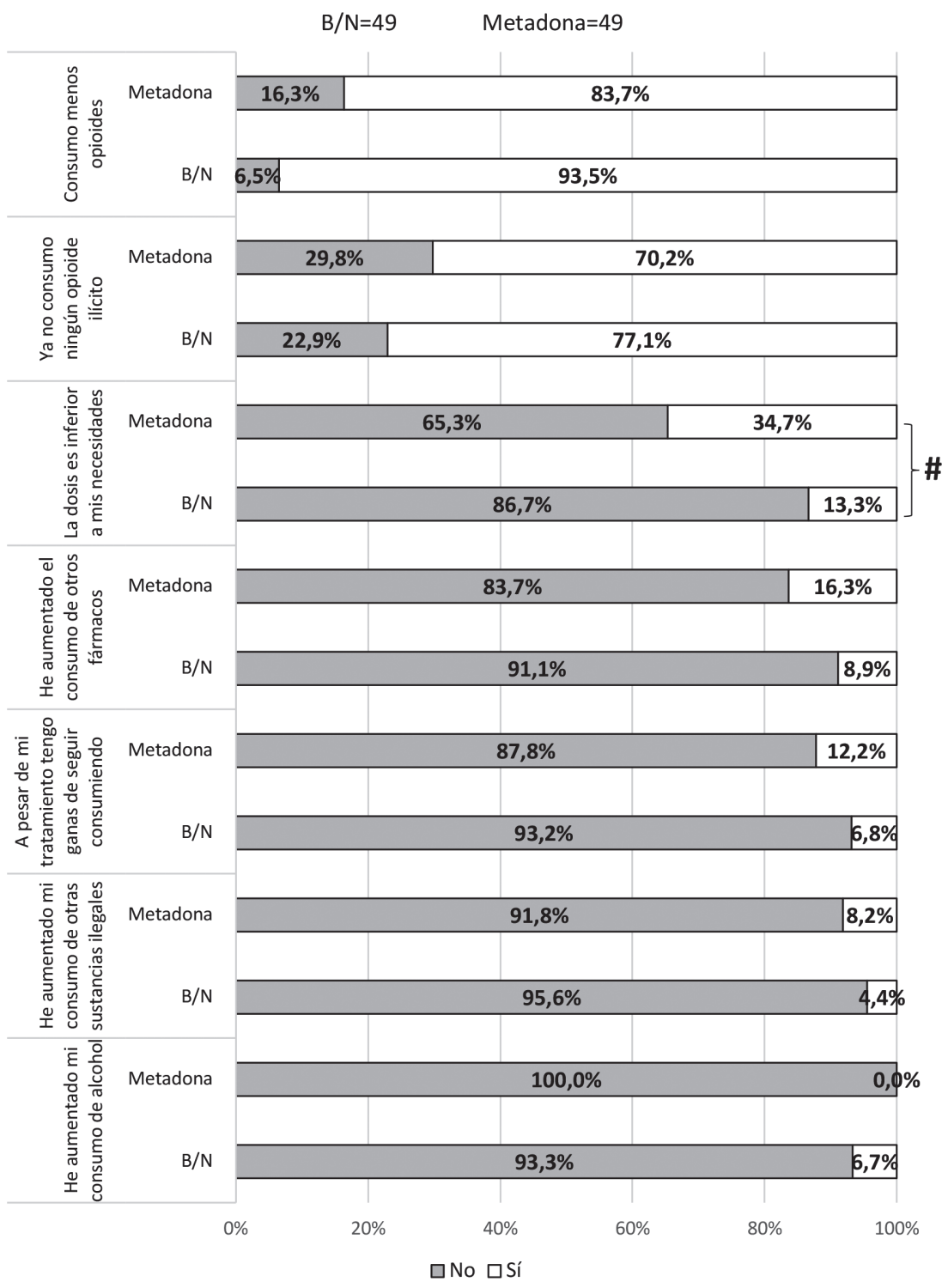

Nota. ${ }^{\#} \mathrm{p}=, 016 ; \mathrm{B} / \mathrm{N}$ : buprenorfina/naloxona; TSO, tratamiento sustitutivo de opioides.

Figura 4. Opinión de los pacientes sobre su TSO.

el motivo de mayor satisfacción fue poder repartir las dosis en varios momentos del día. En conocimiento de los autores, el estudio PREDEPO es el primero en España que evalúa la satisfacción, experiencia, objetivos y opinión de los pacientes con TCO sobre su tratamiento según el TSO prescrito.

Las características sociodemográficas y clínicas siguieron un perfil similar al informado en las estadísticas nacionales para la población con TCO y en TSO en España, sin observarse grandes diferencias entre grupos de tratamiento.

Parece haber una alta satisfacción con el TSO recibido y una alta valoración de su eficacia en ambos grupos, con una puntuación mayor de 8/10 en ambos casos. Sin embargo, en base a las respuestas de los pacientes, se evidencia una falta de satisfacción con ciertos aspectos. Aproximadamente entre el $20 \%$ y el $50 \%$ de los pacientes en ambos grupos in- dicaron que sus TSO actuales no satisfacen sus expectativas por el hecho de tener que recoger diaria o frecuentemente la medicación (lo que además les produce vergüenza o les hace sentir estigmatización asociada al tratamiento), la posibilidad de hacer un mal uso de la medicación, poder de vez en cuando hacer uso de otras sustancias ilícitas y tener que pensar en tomar la medicación diariamente. Más del $50 \%$ de los pacientes con metadona mostraron su insatisfacción con el hecho de tener que recoger la medicación diariamente (más del doble de pacientes en comparación con los pacientes con $\mathrm{B} / \mathrm{N}$ ). Esta diferencia estadísticamente significativa era de esperar, ya que un número significativamente mayor de pacientes con metadona $(89,9 \%)$ recogían la medicación con una frecuencia menor a un mes, en comparación con los pacientes con $\mathrm{B} / \mathrm{N}(23,4 \%)$. 
La necesidad de recoger la medicación o tener que tomar diariamente la medicación es un proceso estigmatizante para los pacientes en TSO. Esto ha sido reportado en otros estudios en los que los pacientes alegan que el proceso de recogida frecuente y dosificación diaria de la medicación es estigmatizante y, por ello, demandan una dosificación del tratamiento menos frecuente, que les permita mayor tiempo libre para normalizar su vida tanto personal como profesional (Harris et al., 2012; Neale, Tompkins, McDonald y Strang, 2018; Treloar et al., 2013). La dispensación frecuente con horarios rígidos, complicados de conciliar con el horario laboral y la dispensación de tratamientos en centros de salud o en unidades de conductas adictivas, en los que coexisten pacientes con diferente grado de adicción, serían factores negativos para pacientes que se encuentran estabilizados (Socidrogalcohol, 2018). En un estudio sobre las opiniones de los pacientes sobre distintas formulaciones de TSO, los participantes consideraron que reducir la carga del tratamiento (tanto en la dispensación como en la administración) supondría una serie de beneficios indirectos como la reducción del estigma, la mejora de la calidad de vida y el aumento del tiempo disponible para completar otras actividades de la vida (Gilman et al., 2018).

Una limitación importante de los TSO actuales, los cuales requieren de una administración diaria, es la posibilidad de vender la medicación o no tomar la medicación para poder consumir sustancias ilícitas de vez en cuando, lo que se traduce en una mala adherencia al tratamiento y un riesgo de recaída (Socidrogalcohol, 2018). En este estudio, en torno al $50 \%$ de los pacientes tratados con $\mathrm{B} / \mathrm{N}$ no les gusta el hecho de tener la posibilidad de desviar su TSO o poder de vez en cuando dejar de tomar la medicación para poder consumir sustancias ilícitas. La necesidad de una administración diaria con los TSO actuales, sumado a la naturaleza del propio trastorno de la adicción, potencia y aumenta la posibilidad de desvío del medicamento al tráfico ilícito o a un consumo indebido y, en consecuencia, a un mayor número de recaídas (Socidrogalcohol, 2018).

Los pacientes en ambos grupos manifestaron una alta predisposición a dejar el consumo de opioides, incluido el TSO ( $74 \%$ con B/N vs. $88 \%$ con metadona). En cuanto a los objetivos de los pacientes con su tratamiento, estos reportaron la necesidad de no tener síndrome de abstinencia, disminuir el consumo de drogas y mejorar su salud. Además, una alta proporción de pacientes refieren querer -no tener más problemas de dinero- $(72 \%$ con $\mathrm{B} / \mathrm{N}$ vs. 92\% con metadona). Todo lo anterior está en línea con lo reportado en un reciente Consenso Europeo de Expertos donde recomiendan que mejorar la salud física y mental, el bienestar y limitar el daño social o económico, asociado con el uso de drogas ilícitas para el individuo y la sociedad, formen parte de los objetivos de tratamiento en estos pacientes (Dematteis et al., 2017).
La mayoría de los pacientes reportaron no olvidarse de tomar la medicación, tomar la dosis prescrita y no encontrar dificultades para su toma. Sin embargo, esto contrasta con los datos publicados de falta de adherencia en pacientes con TCO y el alto porcentaje de abandono del tratamiento, el cual oscila entre el $23 \%$ y el $50 \%$ de pacientes tratados en centros ambulatorios en un periodo de al menos 4 meses en pacientes graves dependientes de opioides que no habían respondido a al menos 4 meses de tratamiento de mantenimiento con metadona (McHugh et al., 2013) y del $17 \%$ al $57 \%$ en centros residenciales en un periodo de 9 meses en pacientes con diagnóstico a lo largo de la vida de abuso o dependencia de sustancias del DSMIV (Samuel, LaPaglia, Maccarelli, Moore y Ball, 2011).

Según estos resultados, los pacientes demandan no sentir más síndrome de abstinencia, reducir el número de visitas a los centros y la necesidad de tomar la medicación todos los días, dejar de pensar en consumir todos los días, reducir el riesgo de desviar la medicación y de tener la opción de no tomar la medicación para seguir consumiendo de vez en cuando, y poder dejar de tener problemas de dinero. Las barreras asociadas a los tratamientos actuales para el TCO, junto con la mala adherencia a los medicamentos existentes, sugiere la necesidad de nuevos tipos de tratamientos que ofrezcan ventajas frente a las terapias actuales que reduzcan la carga del tratamiento y eviten el desvío y el mal uso, proporcionando otra opción terapéutica y aumentando la proporción de pacientes a iniciar un tratamiento que coincida con sus expectativas (Vorspan et al., 2019).

Como fortalezas del presente estudio, cabe destacar que se ha llevado en un contexto de práctica clínica real, y con una muestra con amplia representatividad geográfica de 6 Comunidades Autónomas en España. Por otra parte, este proyecto tiene una serie de limitaciones que se deben tener en cuenta. En el estudio se ha empleado un cuestionario diseñado de forma específica para este estudio basado en el publicado por Rolland et al. (2021). No se consideraron otros cuestionarios validados como el de Pérez de los Cobos et al. (2020) debido a que es una población muy compleja y entendiendo por parte de los autores que un cuestionario con diseño específico podría captar mejor la información de estudio. Sería interesante para investigaciones posteriores realizar una validación psicométrica del cuestionario empleado para valorar sus posibles aplicaciones en otros estudios. Determinó la adherencia terapéutica de los participantes, que hubiera permitido establecer un control de los resultados en función de esta y podría haber aportado información adicional. A pesar de que se alcanzó el tamaño muestral estimado, puede que en alguna de las variables analizadas no se observasen diferencias significativas ente los grupos de tratamiento debido a un reducido tamaño muestral. No se realizaron comparaciones múltiples por procedimientos post-hoc en el caso de ANOVA para intentar reducir el número de comparaciones. Dado el ca- 
rácter exploratorio o generador de hipótesis del estudio se pensó que era la opción más acertada para aclarar objetivos de futuras investigaciones. La selección de los pacientes no se realizó de forma aleatoria lo que hubiera aportado más evidencia a los resultados del estudio. Se optó por una selección consecutiva por entender que era más práctico para la recolección de datos debido a los diferentes periodos de recogida de medicación y a la situación de emergencia derivada de la pandemia de COVID-19.

\section{Conclusión}

En base a las experiencias de los pacientes, parece que existe la necesidad de nuevos tratamientos que aborden las expectativas no cubiertas e insatisfacciones con los tratamientos disponibles actualmente. Los pacientes reportan la necesidad de tratamientos que disminuyan la estigmatización y eviten la necesidad de una administración diaria, lo que podría potencialmente mejorar el manejo del paciente, su adherencia al tratamiento y su calidad de vida.

\section{Reconocimientos}

Los autores agradecen a Ruth Olmos y Hugo López su participación en la organización para el inicio del estudio y a José Joaquín Mira y Miguel Velázquez Gorsse su participación y colaboración en el diseño del estudio.

\section{Conflicto de intereses}

Para el desarrollo de este proyecto, Pharmacoeconomics \& Outcomes Research Iberia (PORIB), una consultora independiente especializada en Evaluación de Intervenciones Sanitarias ha recibido financiación no condicionada a resultados, por parte de Camurus S.L. Carla Assaf Balut es una empleada de Camurus S.L, España.

Los autores Rodrigo Oraá Gil, Gerardo Flórez Menéndez, Pilar Notario Poves, Pedro Seijo Ceballos, Begoña Gonzalvo Cirac y Francisco Pascual Pastor declaran que no tienen conflicto de intereses.

\section{Referencias}

Alonso-Caballero, J. y Ferrer-Fóres, M. M. (2017). Resultados reportados por los pacientes (PROs). Recuperado de https://fundaciongasparcasal.org/wp-content/ uploads/2021/02/Monografia-4-Politica_Resultados-reportados-por-los-pacientes.pdf.

American Psychiatric Association. (2013). Diagnostic and statistical manual of mental disorders: DSM-5. 5th ed. Washington, DC: APA.

Bendimerad, P., Kosim, M. y Trojak, B. (2019, octubre). Patient acceptance and acceptability of a new long-acting form of buprenorphine - AMBRE survey results. Póster presentado en la 14th International Congress of Addiction, Hepatitis and AIDS, Biarritz.

Calvo, F., Carbonell, X., Valero, R., Costa, J., Turró, O., Giralt, C. y Ramírez, M. (2018). Abandono precoz y retención en servicios ambulatorios de drogodependencias: Análisis transversal comparativo de factores que aumentan o disminuyen la adherencia. Atención Primaria, 50, 477-485. doi:10.1016/j.aprim.2017.06.006.

Canadian Agency for Drugs and Technologies in Health. (2013). Rapid response reports. Suboxone ${ }^{\circledR}$ versus methadone for the treatment of opioid dependence: A review of the clinical and cost-effectiveness. Recuperado de http://www.ncbi. nlm.nih.gov/books/NBK195153/.

Dematteis, M., Auriacombe, M., D’Agnone, O., Somaini, L., Szerman, N., Littlewood, R.,... Soyka, M. (2017). Recommendations for buprenorphine and methadone therapy in opioid use disorder: A European consensus. Expert Opinion on Pharmacotherapy, 18, 1987-1999. doi:10.1080/146 56566.2017.1409722.

Florek, A. G., Wang, C. J. y Armstrong, A. W. (2018). Treatment preferences and treatment satisfaction among psoriasis patients: A systematic review. Archives of Dermatological Research, 310, 271-319. doi:10.1007/s00403-0181808-x.

Gilman, M., Li, L., Hudson, K., Lumley, T., Myers, G., Corte, C. y Littlewood, R. (2018). Current and future options for opioid use disorder: A survey assessing real-world opinion of service users on novel therapies including depot formulations of buprenorphine. Patient Preference and Adherence, 12, 2123-2129. doi:10.2147/PPA.S180641.

González-Saiz, F., Lozano Rojas, Ó. M., Martín Esteban, J., Bilbao Acedos, I., Ballesta Gómez, R. y Gutiérrez Ortega, J. (2011). Psychiatric comorbidity in a sample of opiate-dependent patients treated with sublingual buprenorphine in a therapeutic community regime. Revista de Psiquiatría y Salud Mental, 4, 81-87. doi:10.1016/S21735050(11)70013-9.

Harris, J. y McElrath, K. (2012). Methadone as social control: Institutionalized stigma and the prospect of recovery. Qualitative Health Research, 22, 810-824. doi:10.1177/1049732311432718.

Koehl, J. L., Zimmerman, D. E. y Bridgeman, P. J. (2019). Medications for management of opioid use disorder. American Journal of Health-System Pharmacy, 76, 1097-1103. doi:10.1093/ajhp/zxz105.

Iftikhar, A., Allah, N., Shadiullah, K., Habibullah, K., Muhammad, A. R. y Muhammad, H. K. (2011). Predictors of patient satisfaction. Gomal Journal of Medical Sciences, 9, 183-188.

Mateu, G., Astasls, M. y Torrens, M. (2005). Comorbilidad psiquiátrica y trastorno por dependencia de opiáceos: Del diagnóstico al tratamiento. Adicciones, 17,111-121.

McHugh, R. K., Murray, H. W., Hearon, B. A., Pratt, E. M., Pollack, M. H., Safren, S. A. y Otto, M. W. (2013). Predic- 
tors of dropout from psychosocial treatment in opioid-dependent outpatients. The American Journal on Addictions, 22, 18-22. doi:10.1111/j.1521-0391.2013.00317.x.

Neale, J., Tompkins, C.N.E., McDonald, R. y Strang, J. (2018). Patient views of opioid pharmacotherapy biodelivery systems: Qualitative study to assist treatment decision making. Experimental and Clinical Psychopharmacology, 26, 570-581. doi:10.1037/pha0000217.

Observatorio Europeo de las Drogas y las Toxicomanías. (2020). Informe Europeo sobre drogas 2020: Cuestiones clave. Luxemburgo: Oficina de publicaciones de la Unión Europea. Recuperado de https://www.emcdda.europa.eu/system/files/publications/6343/20174796_ TD0117699ESN_PDF.pdf.

Observatorio Europeo de las Drogas y las Toxicomanías. (2019). Informe sobre drogas en España 2019. Madrid: Delegación del Gobierno para el Plan Nacional sobre Drogas. Recuperado de https:/ / www.emcdda.europa.eu/system/ files/publications/11353/20190725_InformeDrogasEspana2019_EMCDDA_DGPNSD.pdf.

Observatorio Vasco de Drogodependencias. (2004). Perfil del Drogodependiente que no responde a los programas de mantenimiento con metadona. País Vasco: GABIA. Recuperado de https:/ / www.osakidetza.euskadi.eus/ataria/.

Pérez de Los Cobos, J., Alcaraz, S., Siñol, N., González-Saiz, F., Vergara-Moragues, E., Trujols, J. y Buprenorphine Naloxone Survey Group. (2020). Towards a common metric for assessing heroin-dependent patient satisfaction with medications: Testing methadone and buprenorphine-naloxone. Drug and Alcohol Dependence, 212, 108010. doi:10.1016/j.drugalcdep.2020.108010.

Rolland, B., Trojak, B., Nourredine, M., Bachellier, J., Chappuy, M., Bendimerad, P.,... Brousse, G. (2021). Determinants of interest in extended-released buprenorphine: A survey among 366 French patients treated with buprenorphine or methadone. Drug and Alcohol Dependence, 220, 108492. doi:10.1016/j.drugalcdep.2020.108492.

Roncero, C., Barral, C., Rodríguez-Cintas, L., Pérez-Pazos, J., Martínez-Luna, N., Casas, M.,... Grau-López, L. (2016). Psychiatric comorbidities in opioid-dependent patients undergoing a replacement therapy programme in Spain: The PROTEUS study. Psychiatry Research, 243, 174-181. doi:10.1016/j.psychres.2016.06.024.

Roncero, C., Domínguez-Hernández, R., Díaz, T., Fernández, J. M., Forcada, R., Martínez, J. M.,... Oyagüez, I. (2015). Management of opioid-dependent patients: Comparison of the cost associated with use of buprenorphine/naloxone or methadone, and their interactions with concomitant treatments for infectious or psychiatric comorbidities. Adicciones, 27, 179-189.

Roncero, C., Fuster, D., Palma-Álvarez, R. F., Rodríguez-Cintas, L., Martínez-Luna, N. y Álvarez, F. J. (2017). HIV And HCV infection among opiate-dependent patients and methadone doses: The PROTEUS study. AIDS Care, 29, 1551-1556. doi:10.1080/09540121.2017.1313384.

Salazar, A., Moreno, S., De Sola, H., Moral-Muñoz, J. A., Dueñas, M. y Failde, I. (2020). The evolution of opioid-related mortality and potential years of life lost in Spain from 2008 to 2017: Differences between Spain and the United States. Current Medical Research and Opinion, 36, 285-291. doi:10.1080/03007995.2019.168425.

Samuel, D. B., LaPaglia, D. M., Maccarelli, L. M., Moore, B. A. y Ball, S. A. (2011). Personality disorders and retention in a therapeutic community for substance dependence. The American Journal on Addictions, 20, 555-562. doi:10.1111/j.1521-0391.2011.00174.x.

Sordo, L., Barrio, G., Bravo, M. J., Indave, B. I., Degenhardt, L., Wiessing, L.,... Pastor-Barriuso, R. (2017). Mortality risk during and after opioid substitution treatment: Systematic review and meta-analysis of cohort studies. BMJ, 357, 1550. doi:10.1136/bmj.j1550.

Socidrogalcohol. (2018). Guía de adicciones para especialistas en formación. Valencia: Socidrogalcohol. Recuperado de https://socidrogalcohol.org/wp-content/Pdf/publicaciones/manuales-guias/guia-adicciones-para-especialistas.pdf.

Strang, J., Reed, K., Bogdanowicz, K., Bell, J., van der Waal, R., Keen, J.,... Knight, A. (2017). Randomised comparison of a novel buprenorphine oral lyophilisate versus existing buprenorphine sublingual tablets in opioid-dependent patients: A first-in-patient phase II randomised open label safety study. European Addiction Research, 23, 61-70. doi:10.1159/000456612.

Treloar, C. y Valentine, K. (2013). Examining structural violence in opioid pharmacotherapy treatment in Australia: Sweating the "small stuff" in a liberal paradise. The International Journal on Drug Policy, 24, 11-13. doi:10.1016/j. drugpo.2013.04.010.

Volkow, N. D., Frieden, T. R., Hyde, P. S. y Cha, S. S. (2014). Medication-assisted therapies-tackling the opioid-overdose epidemic. The New England Journal of Medicine, 370, 2063-2066. doi:10.1056/NEJMp1402780.

Vorspan, F., Hjelmström, P., Simon, N., Benyamina, A., Dervaux, A., Brousse, G.,... Rolland, B. (2019). What place for prolonged-release buprenorphine depot-formulation Buvidal ${ }^{\circledR}$ in the treatment arsenal of opioid dependence? Insights from the French experience on buprenorphine. Expert Opinion on Drug Delivery, 16, 907-914. doi:10.1080/ 17425247.2019.1649252.

World Health Organization. (2018). Information sheet on opioid overdose. Ginebra: World Health Organization. Recuperado de https://www.who.int/substance_abuse/information-sheet/en/. 